\title{
K48-linked ubiquitination and protein degradation regulate 53BP1 recruitment at DNA damage sites
}

\author{
Frédérick A Mallette ${ }^{1,2}$, Stéphane Richard ${ }^{1,2}$ \\ ${ }^{I}$ Terry Fox Molecular Oncology Group and the Bloomfield Center for Research on Aging, Sir Mortimer B Davis Jewish General \\ Hospital, Segal Cancer Centre, Lady Davis Institute for Medical Research, 3755 Côte Ste-Catherine Road, Montréal, Québec, H3T \\ 1E2, Canada; ${ }^{2}$ Departments of Medicine and Oncology, McGill University, Montréal, Québec, Canada \\ Cell Research (2012) 22:1221-1223. doi:10.1038/cr.2012.58; published online 10 April 2012
}

Efficient DNA damage sensing and repair is crucial to preserve genomic integrity and failure to detect or repair DNA breaks can cause mutations, contributing to the formation of tumors. One key protein required for mediating DNA repair is the tumor suppressor 53BP1. Recent studies now demonstrate the crucial role of K48-linked ubiquitination and protein degradation for 53BP1 recruitment at sites of DNA damage.

The linking of ubiquitin chains to proteins is a multistep process involving three different types of enzymes. First, the ubiquitin-activating enzyme E1 catalyzes the transfer of ubiquitin to the ubiquitin-conjugating enzyme E2, which finally conjugates the ubiquitin moeities to target proteins with E3 ubiquitin ligases. Ubiquitin possesses seven lysine residues (K6, K11, K27, K29, $\mathrm{K} 33$, K48 and K63) and an N-terminal methionine (M1) that may be utilized to form poly-ubiquitin chains. The various types of linkage are usually associated with different cellular functions, as K48-linked polyubiquitin chains are involved in proteasomal degradation while K63-linked ubiquitination is a docking site for mediating protein-

Correspondence: Stéphane Richard

E-mail: stephane.richard@mcgill.ca protein interactions or conformational changes. While the role of K63-linked ubiquitination in the DNA damage response has been demonstrated [1], the role of K48-polyubiquitin chains and degradation remained unclear until recently.

In the budding yeast Saccharomyces cerevisiae, components of the $19 \mathrm{~S}$ and $20 \mathrm{~S}$ proteasome are recruited to DNA breaks induced by the $\mathrm{HO}$ endonuclease [2] and mutant strains lacking components of the proteasome displayed enhanced radio-sensitivity. In mammalian cells, inhibition of the proteasome causes defects in the recruitment of multiple players of the DNA damage response pathway including phosphorylated ATM, 53BP1, NBS1, BRCA1, FANCD2 and RAD51 [3]. Furthermore, depletion of the proteasomal subunits PSMB3 and PSMD4 inhibits the formation of FANCD2 foci following ionizing radiation [3]. In addition, K48-linked polyubiquitin chains accumulate at sites of DNA damage, suggesting a role for protein degradation during the DNA damage response [4]. However, the molecular mechanisms controlling protein ubiquitination and degradation following DNA damage are still unclear. The recent discovery of the ubiquitination cascade triggered by the RING finger E3 ubiquitin ligase RNF8 uncovers a novel pathway responsible for the recruitment of DNA damage effectors. In the presence of DNA breaks, the PI3-like protein kinase ATM phosphorylates the histone variant $\mathrm{H} 2 \mathrm{AX}$ on serine 139 , creating a docking station for the mediator protein MDC1, which in turn recruits the ubiquitin ligase RNF8 [5-7]. RNF8 then stimulates the K63-linked ubiquitination of histones $\mathrm{H} 2 \mathrm{~A}$ and $\mathrm{H} 2 \mathrm{AX}$ neighboring the DNA break. The RNF168 and HERC2 ubiquitin ligases also facilitate the linking of ubiquitin to $\mathrm{H} 2 \mathrm{~A}$ at damaged sites [8-10]. The ubiquitination cascade mediated by RNF8, RNF168 and HERC2 is responsible for the efficient recruitment of RAP80/BRCA1 and 53BP1 to DNA damage foci. The formation of RAP80 foci is mediated by its ubiquitininteracting motif(UIM), which binds to K63-linked ubiquitin chains associated to $\mathrm{H} 2 \mathrm{~A}$. However, the recruitment of 53BP1 does not seem to be dependent on K63-linked polyubiquitin chains.

The tumor suppressor p53-binding protein 1 (53BP1) is an important mediator of the DNA damage response. $53 \mathrm{BP} 1$ is required for the efficient phosphorylation of $\mathrm{CHK} 2$ and activation of p53 after damage. Furthermore, 53BP1 contributes to DNA repair through non-homologous end-joining [11]. The formation of 53BP1 foci at DNA damage sites necessitates the binding of the dimethylated histone H4 
on lysine 20 (H4K20) via its tandem tudor domain [12]. Throughout the chromatin, dimethylation of H4K20 is relatively abundant in untreated cells. However, 53BP 1 foci are not observed in undamaged cells, suggesting a mechanism preventing the accessibility of dimethylated H4K20. Nucleosome stacking may contribute to bury methylated H4K20, but since nucleosomes are highly dynamic structures, even buried sites become accessible for protein binding. It was shown that the lysine methyltransferase MMSET is recruited to DNA breaks to locally increase the amount of methylated H4K20 [13]. However, the MMSET-mediated methylation of H4K20 or its localization to DNA breaks is not dependent on RNF8 activity [13]. Hence, the local enrichment of $\mathrm{H} 4 \mathrm{~K} 20$ (me2) triggered by MMSET does not explain the requirement of RNF8 for the recruitment of 53BP1 [5-7]. It was initially thought that RNF8 only catalyzes K63-linked ubiquitination, but multiple recent reports describe the ability of RNF8 to stimulate the formation of K48-linked ubiquitin chains $[4,14,15]$, confirming a role for RNF8-mediated degradation or chromatin extraction during the DNA damage response.

We identified the lysine demethylases JMJD2A and JMJD2B as novel targets of RNF8-mediated K48-linked ubiquitination (Figure 1) [15]. Both JMJD2A and JMJD2B possess a hybrid tandem tudor domain conferring the ability to bind methylated H4K20. In fact, JMJD2A $\left(\mathrm{K}_{\mathrm{D}}=2.0 \mu \mathrm{M}\right)$ and JMJD2B $\left(\mathrm{K}_{\mathrm{D}}=27.7 \mu \mathrm{M}\right)$ bind dimethylated H4K20 with a higher relative affinity than 53BP1 $\left(\mathrm{K}_{\mathrm{D}}=50.8 \mu \mathrm{M}\right)$ and they can block the formation 53BP1 foci when ectopically expressed [15]. In response to DNA breaks, both RNF8 and RNF168 can directly ubiquitinate JMDJ2A, leading to its proteasomal degradation. Depletion of RNF8 in the cell caused marked defects in the recruitment of 53BP1, but combined depletion of JMJD2A and JMJD2B in
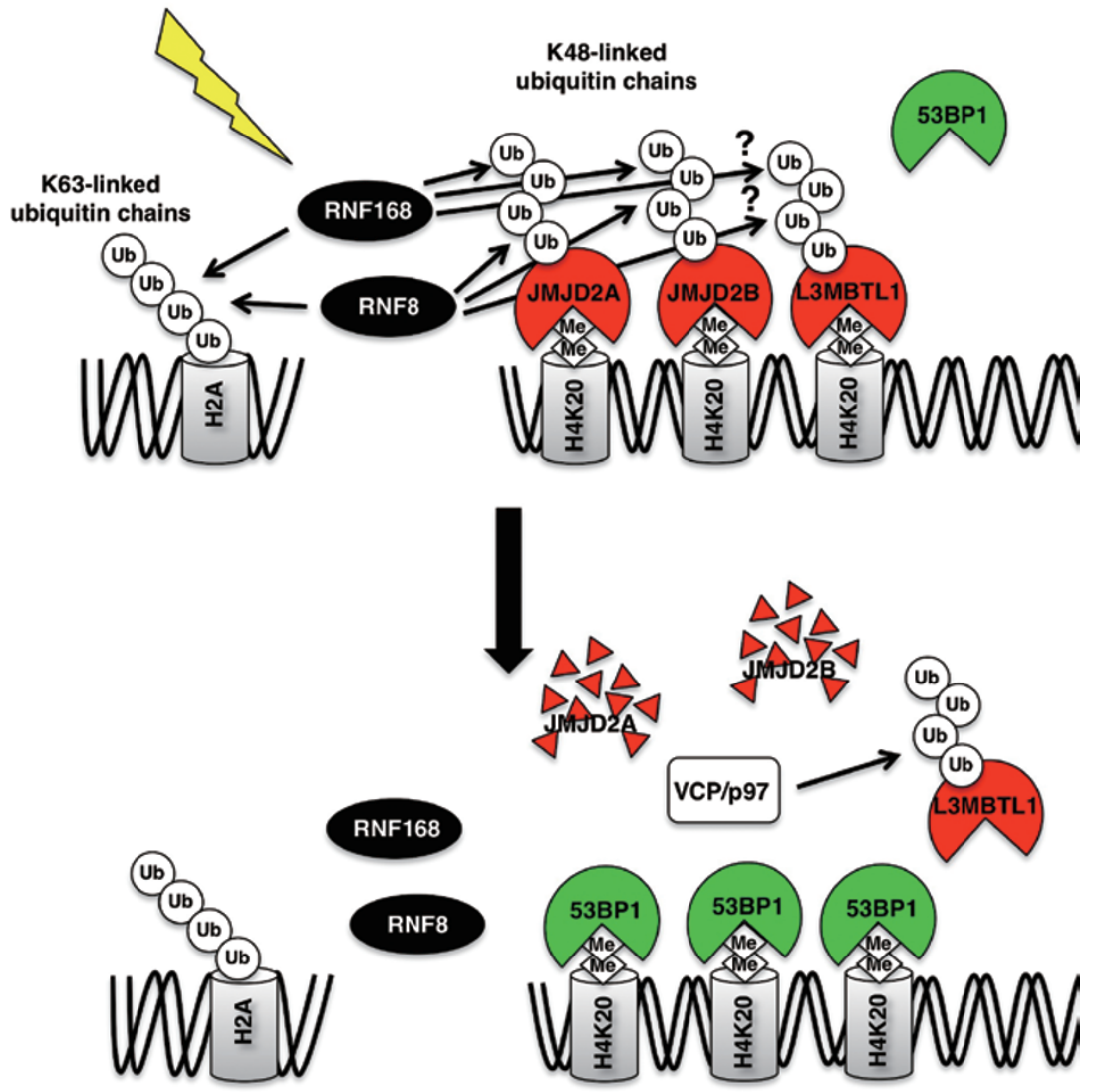

Figure $1 \mathrm{~K} 48$-linked ubiquitination regulates the recruitment of 53BP1 to DNA lesions. JMJD2A, JMJD2B and L3MBTL1 are bound to methylated H4K20 in untreated cells, thus preventing the recruitment of 53BP1. Following DNA damage, the RNF8 and RNF168 ubiquitin ligases are recruited to DNA breaks where they mediate protein ubiquitination. The ubiquitination of L3MBTL1 promotes its chormatin extraction in a VCP/p97- and RNF8/168-dependent manner. Whether L3MBTL1 represents a direct substrate of RNF8 and RNF168 remains to be explored. RNF8- and RNF168-mediated K48-linked ubiquitination of JMJD2A and JMJD2B directs them to the proteasome for degradation, therefore allowing the recruitment of 53BP1 to dimethylated H4K20. This enables the local recruitment of 53BP1 to DNA damage sites.

these cells restores the formation of 53BP1 foci [15]. These results suggest that JMJD2A and JMJD2B are bound to methylated $\mathrm{H} 4 \mathrm{~K} 20$ in untreated cells and that RNF8- and RNF168-dependent activity lead to their chromatin extraction and proteasomal degradation to allow the unmasking of $\mathrm{H} 4 \mathrm{~K} 20$ and binding of 53BP1. In addition to JMJD2A, novel substrates of RNF8dependent degradation, such as KU80 and CHK2, have been recently identified, confirming the important role of proteasomal degradation in the DNA damage response [14].

K48-linked poly-ubiquitination also promotes the chromatin extraction of proteins by the ubiquitin-selective segregase $\mathrm{VCP} / \mathrm{p} 97$. $\mathrm{VCP} / \mathrm{p} 97$ is recruited to DNA breaks in an RNF8-dependent fashion requiring the formation of K48-linked ubiquitin chains [4]. The presence of $\mathrm{VCP} / \mathrm{p} 97$ at DNA lesions is a prerequisite for 53BP1 foci formation and may promote the chromatin extraction of ubiquitinated proteins 
[4]. In fact, the Polycomb group protein L3MBTL1 is removed from DNA lesions by an RNF8- and VCP/p97dependent mechanism [16]. L3MBTL1 possess three MBT domains allowing its binding to methylated H4K20 and potentially competes with 53BP1 for this methylated histone mark at DNA damaged sites. The chromatin extraction of ubiquitinated L3MBTL1 correlates with the local recruitment of 53BP1 [16]. The affinity of L3MBTL1 $\left(\mathrm{K}_{\mathrm{D}}=211 \mu \mathrm{M}\right.$ [17]) for dimethylated H4K20 is relatively low compared to 53BP1 $\left(\mathrm{K}_{\mathrm{D}}=50.8 \mu \mathrm{M}\right)$, suggesting that JMJD2A $\left(\mathrm{K}_{\mathrm{D}}=2.0 \mu \mathrm{M}\right)$ and JMJD2B $\left(K_{D}=27.7 \mu \mathrm{M}\right)$ release from the chromatin is important for the formation of 53BP1 foci. The K48-linked ubiquitination of JMJD2A and JMJD2B by RNF8 and RNF168 might also promote their $\mathrm{VCP} / \mathrm{p} 97-$ dependent chromatin extraction from DNA breaks as suggested by their rapid and massive dissociation from the chromatin [15].

Together, these recent reports elucidate the molecular mechanism underlying the recruitment of 53BP1 to DNA breaks and reconcile the previous models by unifying RNF8-mediated ubiquitination, proteasomal degradation and the role of methylation of histone $\mathrm{H} 4$ on lysine 20. In conclusion, proteins possessing tandem tudor domains, such as JMJD2A, JMJD2B and the MBT-containing L3MBTL1, are masking the methylated H4K20 mark and are extracted from the chromatin and degraded to allow the recruitment of 53BP1 to DNA lesions.

\section{Acknowledgments}

We thank Gillian Vogel and members of the Richard laboratory for helpful comments. FAM received postdoctoral fellowships from the Canadian Institutes of Health Research (CIHR). This work was funded by CIHR grant MOP67070 to SR.

\section{References}

1 Panier S, Durocher D. Regulatory ubiquitylation in response to DNA doublestrand breaks. DNA Repair (Amst) 2009; 8:436-443.

2 Krogan NJ, Lam MH, Fillingham J, et al. Proteasome involvement in the repair of DNA double-strand breaks. $\mathrm{Mol}$ Cell 2004; 16:1027-1034.

3 Jacquemont C, Taniguchi T. Proteasome function is required for DNA damage response and fanconi anemia pathway activation. Cancer Res 2007; 67:7395-7405.

4 Meerang M, Ritz D, Paliwal S, et al. The ubiquitin-selective segregase $\mathrm{VCP} / \mathrm{p} 97$ orchestrates the response to DNA double-strand breaks. Nat Cell Biol 2011; 13:1376-1382.

5 Huen MS, Grant R, Manke I, et al. RNF8 transduces the DNA-damage signal via histone ubiquitylation and checkpoint protein assembly. Cell 2007; 131:901-914.

6 Kolas NK, Chapman JR, Nakada S, et al. Orchestration of the DNA-damage response by the RNF8 ubiquitin ligase. Science 2007; 318:1637-1640.

7 Mailand N, Bekker-Jensen S, Faustrup $\mathrm{H}$, et al. RNF8 ubiquitylates histones at DNA double-strand breaks and promotes assembly of repair proteins. Cell 2007; 131:887-900.

8 Bekker-Jensen S, Danielsen JR, Fugger $\mathrm{K}$, et al. HERC2 coordinates ubiquitindependent assembly of DNA repair factors on damaged chromosomes. Nat Cell Biol 2010; 12:80-86; sup pp 8112.

9 Doil C, Mailand N, Bekker-Jensen S, et al. RNF168 binds and amplifies ubiquitin conjugates on damaged chromosomes to allow accumulation of repair proteins. Cell 2009; 136:435-446.

10 Stewart GS, Panier S, Townsend K, et $a l$. The RIDDLE syndrome protein mediates a ubiquitin-dependent signaling cascade at sites of DNA damage. Cell 2009; 136:420-434.

11 FitzGerald JE, Grenon M, Lowndes NF. 53BP1: function and mechanisms of focal recruitment. Biochem Soc Trans 2009; 37(Pt 4):897-904.

12 Botuyan MV, Lee J, Ward IM, et al. Structural basis for the methylation state-specific recognition of histone H4-K20 by 53BP1 and Crb2 in DNA repair. Cell 2006; 127:1361-1373.

13 Pei H, Zhang L, Luo K, et al. MMSET regulates histone $\mathrm{H} 4 \mathrm{~K} 20$ methylation and 53BP1 accumulation at DNA damage sites. Nature 2011; 470:124-128.

14 Feng L, Chen J. The E3 ligase RNF8 regulates KU80 removal and NHEJ repair. Nat Struct Mol Biol 2012; 19:201206.

15 Mallette FA, Mattiroli F, Cui G, et al. RNF8- and RNF168-dependent degradation of KDM4A/JMJD2A triggers $53 \mathrm{BP} 1$ recruitment to DNA damage sites. EMBO J 2012; 31:1865-1878.

16 Acs K, Luijsterburg MS, Ackermann L, et al. The AAA-ATPase VCP/p97 promotes 53BP1 recruitment by removing L3MBTL1 from DNA double-strand breaks. Nat Struct Mol Biol 2011; 18:1345-1350.

17 Min J, Allali-Hassani A, Nady N, et al. L3MBTL1 recognition of mono- and dimethylated histones. Nat Struct Mol Biol 2007; 14:1229-1230. 